Article

\title{
Game-Theory Based V2G Coordination Strategy for Providing Ramping Flexibility in Power Systems
}

\author{
Jin Zhang ${ }^{1}$, Liang Che ${ }^{1, *}$, Lei Wang ${ }^{1}$ and Udaya K. Madawala ${ }^{2}$ \\ 1 College of Electrical and Information Engineering, Hunan University, Changsha 410082, China; \\ zhangjin1994@hnu.edu.cn (J.Z.); jordanwanglei@hnu.edu.cn (L.W.) \\ 2 Department of Electrical, Computer and Software Engineering, Faculty of Engineering, \\ The University of Auckalnd, Auckalnd 1023, New Zealand; u.madawala@auckland.ac.nz \\ * Correspondence: cheliang@hnu.edu.cn; Tel.: +86-1580-250-0171
}

Received: 24 August 2020; Accepted: 23 September 2020; Published: 23 September 2020

check for updates

\begin{abstract}
Large-scale integration of renewable generation into power systems invariably affects the system ramping capability. However, the vehicle-to-grid (V2G) concept that allows for using electric vehicles (EVs) as energy storages with the capability of bidirectional energy transfer between the EVs and the grid, can be employed to mitigate the above adverse effect. This paper proposes a game-theory-based V2G coordination strategy that uses EV clusters to improve ramping flexibility in power systems. In the proposed strategy, the V2G concept, representing the interactions between the distribution system operator (DSO) and EV clusters, is formulated as a Stackelberg game. The DSO acts as a leader who decides the charging prices for the buses to which the EV clusters are connected, while the EV clusters simply serve as followers, scheduling their own charging and discharging. This bi-level model is further reduced to a single-level, mixed-integer second-order cone programming (MISOCP) problem based on the Karush-Kuhn-Tucker (KKT) conditions, the strong duality theorem and second-order cone (SOC) relaxation. The performance of the proposed V2G coordination strategy on a modified IEEE 33-bus system connecting EV clusters and PV generations is investigated through simulations, and the results demonstrate that the largest ramp of the system can be reduced by up to $39 \%$ when EV clusters are providing flexibility, while the EV clusters can also have greatly reduced charging costs.
\end{abstract}

Keywords: V2G; electric vehicle; Stackelberg game; power market

\section{Introduction}

Power systems have been experiencing a fast increase in renewable energy integration all over the world. It is estimated that by 2050 , solar PV power generation will account for $16 \%$ of the total electricity in the world and $43 \%$ of the added electricity in the United States [1]. However, the increasing penetration of renewable generation in power systems will impose significant challenges to the system operation [2-4]. A main concern is that the intermittent nature of renewables will cause fast ramping and change in the system net load over a short period of time. Reference [5] depicts California's daily net load curves, also known as "duck curves", from 2012 to 2020. Such duck curves highlight the rapid ramping of net demand under high penetration of renewables and illustrates that the system, with increasing integration of renewables, confronts the problem of a shortage of ramping capability, threatening the stable operation of power systems.

A number of researches have investigated strategies to flatten the duck curve, which can be categorized into four types. The first one is to apply renewable generation curtailment as reported in [6-8]. This strategy is easy to implement with small adjustments to the controllers. However, this led to a high curtailment ratio of renewable energy. The second type of strategy, proposed by 
CAISO [5], is to increase the amount of load by means of expanding the ISO's footprint. Apparently, this is just a temporary solution and does not address the issue of the ramping shortage. The third strategy, proposed from the perspective of the supply side, is to enhance the flexibility of generation resources to quickly follow the dispatch for alleviating the system's fluctuations [9-12]. Although it offers an effective way for accommodating more renewable energy, it needs a large investment in adding new plants or retrofitting the existing ones. The fourth type of strategy, as emphasized in this paper, uses demand-side management approaches for addressing the system ramping issue. For example, Reference [13] investigates the contribution of load to system balance and flexibility, where interruptible load is explored as the provider of flexible ramping to the system. Specifically, a load serving entity acts as a mediator who interacts with the system to satisfy ramping needs, while offering incentives to the interruptible loads. Reference [14] employs a dynamic pricing scheme to flatten the duck curve by using the air-conditioners and energy storages of end-users. To address the ramp shortage problem, the optimal energy consumption scheduling for prosumers in a distribution system is studied in [15]. An aggregator is introduced in [16] to integrate a group of small demand response participants into the electricity market for ramping regulation, where the aggregator minimizes its cost by autonomously selecting appropriate programs. These studies have shed light on the demand-side approaches for addressing the ramp shortage issue. However, they have considered neither the system congestion management, nor the bidirectional energy transfer between the participants and the system. In fact, when the distribution system operator (DSO) interacts with distinct entities for ramping requirements, the network constraints will have a decisive impact on its decision-making for the system's secure operation.

With the recent advances in vehicle-to-grid (V2G) technology, a cluster of electric vehicles (EVs), with controlled charging and discharging, can act as an energy storage to improve the ramping flexibility of the power system that experiences the aforementioned ramping shortage issue under high renewable penetration scenarios.

EVs' participation in demand response has been studied in [17]. EV batteries have a much faster ramp rate than conventional generations for regulation service provision [18]. With the application of V2G technology, EV clusters would play a crucial role in system frequency regulation $[19,20]$, voltage regulation [21,22], and ramping regulation [23-26]. Considering the variability of wind power, Reference [23] proposes a hierarchical scheme to control EVs to address the problem of wind-induced generator ramp cycling. To investigate the impact of EVs' participation in providing ramping, Reference [24] compares EV-provided ramping with that provided by EVs in collaboration with conventional generators. Reference [25] explores the potential of dispatching EVs to participate in the ramp market though a locational marginal pricing-based model. Reference [26] studies the effect of PV penetration on the Singapore power grid and uses EVs to support reducing the ramping cost of the system. However, References [23-26] do not consider the interactions between the system operator and EVs. In fact, the DSO and EVs have their own objectives and their operational decisions may impact the other's objectives. Therefore, it is necessary to investigate the interaction between the DSO and the clustered EVs with V2G technology for studying how the EV clusters can contribute ramping flexibility to the power system. To the best of our knowledge, this aspect has not been investigated in detail, according to the literature.

This paper therefore proposes a game-theory-based V2G coordination strategy for the EV clusters to improve the ramping flexibility of power systems that are subjected to variable renewable penetrations. The main contributions of this paper are summarized below:

1. We propose to use the EV clusters as a flexible resource to improve system ramping capability while fully considering the power flow congestion in the power network.

2. In order to study $\mathrm{V} 2 \mathrm{G}$ coordination, we propose a dynamic pricing mechanism, which models the DSO as a leader deciding the electricity trading prices at the EV-cluster-connected buses, and models the EVs as followers responding to the prices by scheduling their V2G charging and discharging. 
3. The Stackelberg-game-based bi-level model that we propose is reformulated as a single-level mixed-integer second-order cone programming (MISOCP) problem by using Karush-Kuhn-Tucker (KKT) conditions, the strong duality theorem and second-order cone (SOC) relaxation, and thus can be efficiently solved by commercial solvers.

\section{System Model and the Proposed Strategy}

\subsection{Active Distribution System}

In this paper, we consider an active distribution system connecting multiple EV clusters as shown

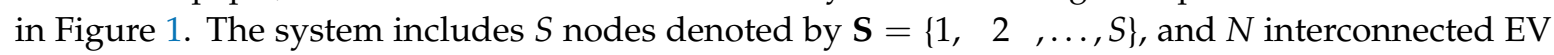
clusters denoted by $\mathbf{N}=\{1,2, \ldots, N\}$. Each EV cluster coordinates $M$ number of EVs denoted by $\mathbf{M}=\{1,2, \ldots, M\}$. The studied operational time horizon is one day, which is divided into $T=24 \mathrm{~h}$ and represented by the set $\mathbf{T}=\{1,2, \ldots, T\}$.

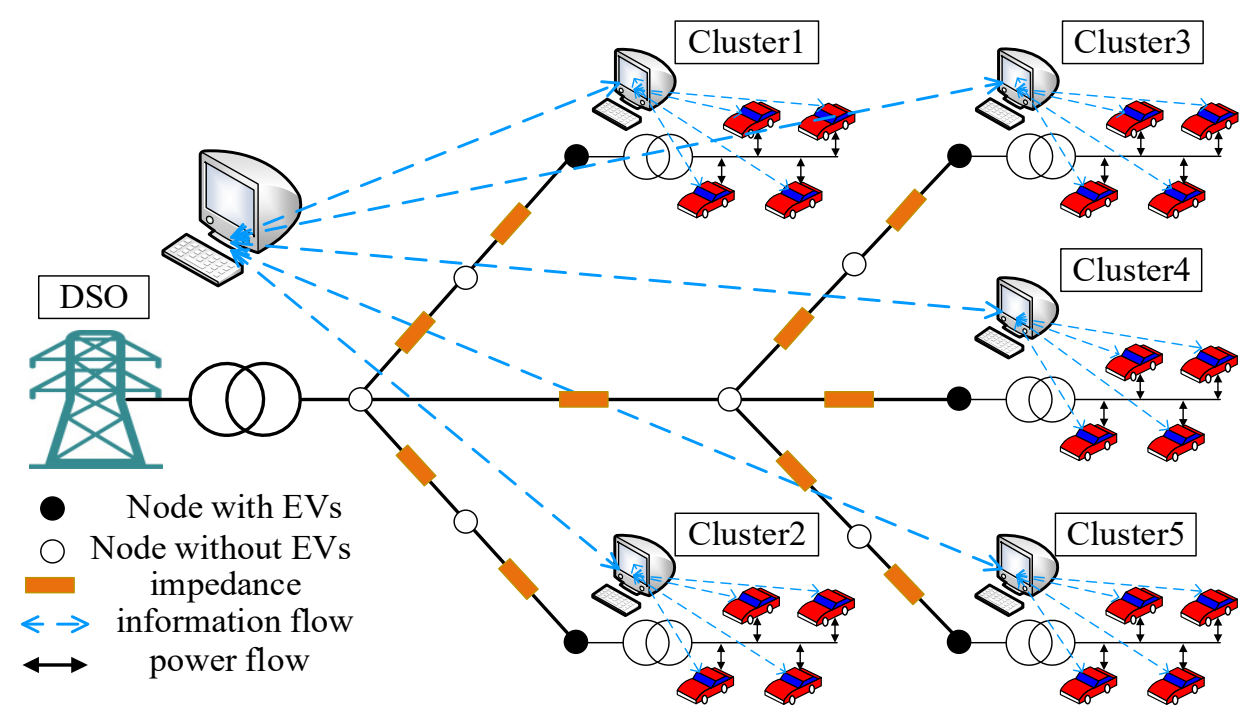

Figure 1. Schematic of the active distribution system with multiple electric vehicle (EV) clusters.

In practical system operations, the direct participation of the EVs into the demand-side management program is generally not allowed due to the low energy capacities of individual EVs. For example, in ISO-NE, the minimum capacity for providing regulation service is $1 \mathrm{MW}$, but the capacity of a single EV is generally less than $100 \mathrm{~kW}$ [27]. Therefore, there is a need for an agent to coordinate a cluster of EVs to meet the minimum capacity requirement as depicted in Figure 1. The agent managing an EV cluster is a third-party entity, such as a charging station owner, who has a competitive relationship with the DSO in the deregulated electricity market. In the system, V2G technology and the two-way communication infrastructure allow the bidirectional flows of power and information between the distribution system and the EV clusters.

\subsection{Proposed Stackelberg-Game-Based Coordination Strategy}

Before presenting the proposed strategy, it is necessary to analyze the interactions between the DSO and the EV clusters. Apparently, the EVs' charging and discharging behaviors are mainly driven by economic factors as each EV is essentially a rational economic entity. The DSO needs the EVs to provide ramp flexibilities. To this end, the DSO needs to offer economic incentives to the EV owners, who will consider the degradation of their batteries. Such an interaction between the DSO and the EV clusters constitutes a Stackelberg game, in which the DSO sets the transaction price to guide the EVs' charging and discharging, i.e., the provision of flexibility. The game is illustrated in Figure 2, and the detailed descriptions of each participant in the game are given as follows: 


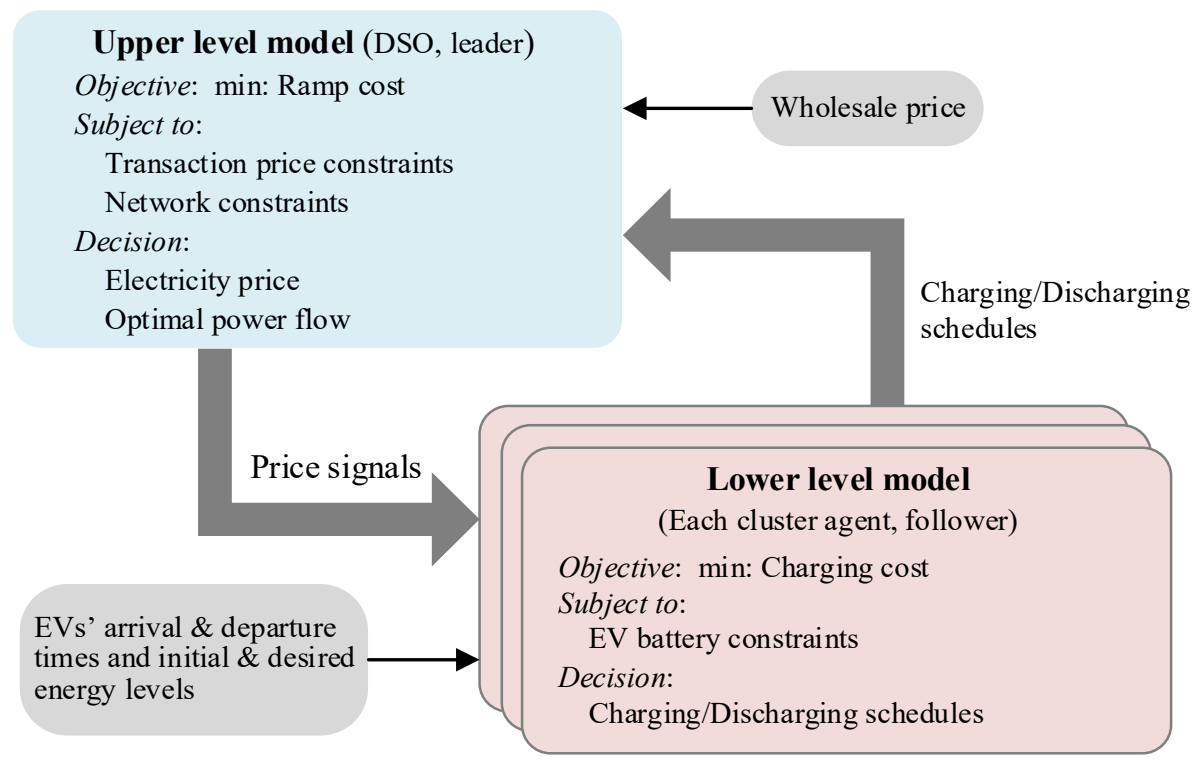

Figure 2. Stackelberg game between the distribution system operator (DSO) and the EV clusters.

DSO: The DSO acts as a leader to encourage the EVs to provide ramping flexibility. Based on the forecasted energy prices and ramping request in the next operation horizon, the DSO determines a set of electricity prices as incentives to the EV clusters. Considering the locations of the clusters in the distribution grid, the DSO sets dynamic transaction prices:

$$
\begin{aligned}
& \pi^{n}=\left\{\pi_{1}^{n}, \pi_{2}^{n}, \ldots \pi_{T}^{n}\right\} \quad \forall n \\
& \pi_{t}^{n, \min } \leq \pi_{t}^{n} \leq \pi_{t}^{n, \max } \quad \forall n, \forall t,
\end{aligned}
$$

where $\pi^{n}$ denotes the charging and discharging price designed by DSO for the EV cluster agent $n$ in the operational time horizon, $\pi_{t}^{n}$ represents the transaction prices in time slot $t$, and $\pi_{t}^{n, \min }$ and $\pi_{t}^{n, \max }$ denote the lower and upper bounds of the transaction prices, respectively.

EV cluster agent: The EV cluster agent is responsible for coordinating the EVs in the game. The agent is equipped with a database for storing its EVs' data, including arrival and departure times, the initial and desired energy levels, etc., which could be uploaded by the EV owners upon arrival at the charging station. In fact, the EV owners may have privacy considerations when sharing such data with the agent, but the privacy issue is out of the scope of this paper and is left for future research. The ramping discussed in this paper is provided in the day-ahead power market. In the market, a cluster agent will forecast the number and the operational data of the EVs during the next operating time period based on the historical information in its database, and by using the forecasted information, the cluster agent will play the game with the DSO. In the game, the cluster agent acts as a follower who minimizes the total charging costs of its clustered (managed) EVs by scheduling their charging and discharging, based on the transaction prices given by the DSO.

The Stackelberg game discussed above forms the basis for the proposed coordination strategy in this paper.

\section{Mathematical Formulation}

As discussed in the previous section, the proposed coordination strategy is built on a Stackelberg game among the DSO and multiple EV clusters. In this section, we provide the mathematical model of this game. In the following, the game is formulated as a bilevel model, and is then reformulated as a single-level MISOCP problem that can be efficiently solved using commercial optimization solvers. 


\subsection{Model of DSO}

Let $P_{t}^{r e}$ and $P_{t}^{\text {load }}$ be the total renewable generation and the total load in the distribution grid at time slot $t$. So, the system net load (without considering the EV clusters) at time slot $t$ is

$$
\delta_{t}=P_{t}^{\text {load }}-P_{t}^{r e} \quad \forall t .
$$

When multiple EV clusters are participating in providing the ramping flexibility, the ramping of the net load between two consecutive time slots and the ramp vector during the entire time horizon are given in (4) and (5), respectively:

$$
\begin{gathered}
r_{t}=\delta_{t}-\delta_{t-1}+\sum_{n=1}^{N} \sum_{m=1}^{M}\left[\left(E_{t}^{m, n}-e_{t}^{m, n}\right)-\left(E_{t-1}^{m, n}-e_{t-1}^{m, n}\right)\right] \quad \forall t \\
\mathbf{R}=\left\{r_{1}, r_{2}, \cdots r_{T}\right\}
\end{gathered}
$$

where $E_{t}^{m, n}$ and $e_{t}^{m, n}$ respectively denote the charging and discharging powers of EV $m$ coordinated by cluster agent $n$.

Therefore, the largest ramp of the system net load during the time horizon is

$$
\|\mathbf{R}\|_{\infty}=\max \left\{|r|_{1},\left|r_{2}\right|, \cdots|r|_{T}\right\}
$$

where $\|\cdot\|_{\infty}$ represents the infinity-norm.

In the Stackelberg game shown in Figure 2, the DSO aims at minimizing the cost of obtaining the ramp flexibility from all participating EV clusters. So, according to (1)-(6), the DSO's objective function is:

$$
\min \left[a\left(\|\mathbf{R}\|_{\infty}\right)^{2}+b\|\mathbf{R}\|_{\infty}\right]-\sum_{n=1}^{N} \sum_{m=1}^{M} \sum_{t=1}^{T} \pi_{t}^{n}\left(E_{t}^{m, n}-e_{t}^{m, n}\right),
$$

where the first term denotes the cost of the ramp with a quadratic function according to [28], $a$ and $b$ are the positive cost coefficients, and the second term is the revenue obtained from the transaction with the EVs. Since $\pi_{t}^{n}, E_{t}^{m, n}$ and $e_{t}^{m, n}$ are decision variables, the objective function (7) is neither convex nor linear.

From (7), it can be seen that the appropriate transaction prices $\pi_{t}^{n}$ set by the DSO will address the ramp shortage issue due to the fact that it will influence the participating EVs' charging and discharging behaviors.

Next, we present how the power flow congestion in the distribution grid is modeled in the game. A distribution grid is typically a radial power network, as shown in Figure 1. The power flows of a radial network can be solved by the distflow branch model [29], as given below:

$$
\begin{gathered}
P_{t}^{i j}-\left(I_{t}^{i j}\right)^{2} R_{i j}=\sum_{k \in \omega(j)} P_{t}^{j k}+P_{t}^{j} \quad \forall t, \forall i, j \in \mathbf{S} \\
Q_{t}^{i j}-\left(I_{t}^{i j}\right)^{2} X_{i j}=\sum_{k \in \omega(j)} Q_{t}^{j k}+Q_{t}^{j} \quad \forall t, \forall i, j \in \mathbf{S} \\
\left(V_{t}^{j}\right)^{2}=\left(V_{t}^{i}\right)^{2}-2\left(R_{i j} P_{t}^{i j}+X_{i j} Q_{t}^{i j}\right)+\left(I_{t}^{i j}\right)^{2}\left(R_{i j}^{2}+X_{i j}^{2}\right) \quad \forall t, \forall i, j \in \mathbf{S} \\
\left(I_{t}^{i j}\right)^{2}=\frac{\left(P_{t}^{i j}\right)^{2}+\left(Q_{t}^{i j}\right)^{2}}{\left(V_{t}^{i}\right)^{2}} \quad \forall t, \forall i, j \in \mathbf{S} \\
v_{t}^{i, \min } \leq\left(V_{t}^{i}\right)^{2} \leq v_{t}^{i, \max } \quad \forall t, \forall i, j \in \mathbf{S}
\end{gathered}
$$




$$
0<\left(I_{t}^{i j}\right)^{2} \leq i_{t}^{i j, \max } \quad \forall t, \forall i, j \in \mathbf{S},
$$

where $\omega(j)$ is the set of bus indices that are connected to bus $j ; R_{i j}$ and $X_{i j}$ are branch $i j$ 's resistance and reactance, respectively; $P_{t}^{i j}$ and $Q_{t}^{i j}$ respectively denote branch $i j^{\prime}$ s active and reactive power flows; $P_{t}^{j}$ and $Q_{t}^{j}$ denote the active and reactive load of bus $j ; V_{t}^{i}, v_{t}^{i, \min }$ and $v_{t}^{i, \max }$ denote the voltage and the minimal and maximal squares of the voltages, respectively, of bus $I$; and $I_{t}^{i j}$ and $i_{t}^{i j, m a x}$ respectively denote the current and the maximal square of the current of branch $i j$.

\subsection{Model of an EV Cluster}

As a follower in the Stackelberg game, each EV cluster agent aims at minimizing the total charging and discharging cost of its clustered EVs. Therefore, the objective function of an EV cluster $n$ is:

$$
\min \sum_{m=1}^{M} \sum_{t=1}^{T} \pi_{t}^{n}\left(E_{t}^{m, n}-e_{t}^{m, n}\right)
$$

In terms of the EVs' charging powers, the EV cluster agent should consider the following constraints for its clustered EVs:

$$
\begin{array}{cc}
0 \leq E_{t}^{n, m} \leq E^{n, m, \max } & t \in\left[t_{\text {in }}^{n, m}, t_{\text {out }}^{n, m}\right], \forall n, \forall m \\
0 \leq e_{t}^{n, m} \leq e^{n, m, \max } & t \in\left[t_{\text {in }}^{n, m}, t_{\text {out }}^{n, m}\right], \forall n, \forall m \\
E_{t}^{n, m}, e_{t}^{n, m}=0 & t \notin\left[t_{\text {in }}^{n, m}, t_{\text {out }}^{n, m}\right], \forall n, \forall m,
\end{array}
$$

where (15) and (16) represent that the charging and discharging power, respectively, which should not exceed the limitations, $E^{n, m, \max }$ and $e^{n, m, \max }$, respectively; and (17) indicates that the EV is not in a charging or discharging status (e.g., the EV has departed from the charging station).

When an EV arrives at the charging station, it will inform the cluster agent about its desired energy level $S^{n, m}$, i.e., the needed energy level when the EV departs from the charging station. This is specified in the following equation:

$$
\sum_{t_{\text {in }}^{n, m}}^{t_{\text {out }}^{n, m}}\left(E_{t}^{n, m}-e_{t}^{n, m}\right)=S^{n, m} \quad \forall n, \forall m .
$$

At the same time, to protect the EV battery from early degradation and to consider the EV battery technology constraints, the energy level of each EV should also satisfy:

$$
S_{n, m}^{\min } \leq S_{o}^{n, m}+\sum_{t_{\text {in }}^{n, m}}^{t}\left(E_{t}^{n, m}-e_{t}^{n, m}\right) \leq S_{n, m}^{\max } \quad t \in\left[t_{\text {in }}^{n, m}, t_{\text {out }}^{n, m}\right], \forall n, \forall m,
$$

where $S_{o}^{n, m}$ denotes the initial battery energy level of EV $m$, and $S_{n, m}^{\min }$ and $S_{n, m}^{\max }$ are the prescribed lower and upper bounds, respectively, of $S_{o}^{n, m}$.

\subsection{Model Reformulation}

From Figure 2, it can be seen that the Stackelberg game between the DSO and the EV clusters is a bilevel problem, which cannot be solved straightforwardly due to the non-linear and non-convex terms, including the bilinear terms $\pi_{t}^{n}\left(E_{t}^{m, n}-e_{t}^{m, n}\right)$ in the upper level objective function and in the non-convex distflow model (8)-(11). To efficiently solve the problem, the original problem is reformulated as a single-level MISOCP problem and the bilinear terms in the objective function (7) are linearized. 


\subsubsection{Linearization of Bilinear Terms}

Since the internal price $\pi_{t}^{n}$ is fixed for the EV owners, the lower level (14)-(19) is linear and continuous. Therefore, we can replace the lower level with its KKT conditions as follows:

Stationary conditions:

$$
\begin{array}{ll}
\pi_{t}^{n}-\mu_{1, t}^{n, m}+\mu_{2, t}^{n, m}-\eta_{1, t}^{n, m}-\zeta^{n, m}-\sum_{t}^{t_{\text {out }}^{n, m}} \mu_{5, t}^{n, m}+\sum_{t}^{t_{\text {out }}^{n, m}} \mu_{6, t}^{n, m}=0 & \forall n, \forall m \\
-\pi_{t}^{n}-\mu_{3, t}^{n, m}+\mu_{4, t}^{n, m}-\eta_{2, t}^{n, m}+\zeta^{n, m}+\sum_{t}^{t_{\text {out }}^{n, m}} \mu_{5, t}^{n, m}-\sum_{t}^{t_{\text {out }}^{n, m}} \mu_{6, t}^{n, m}=0 & \forall n, \forall m .
\end{array}
$$

Complementary slackness conditions:

$$
\begin{aligned}
& 0 \leq E_{t}^{n, m} \perp \mu_{1, t}^{n, m} \geq 0 \quad t \in\left[t_{\text {in }}^{n, m}, t_{\text {out }}^{n, m}\right], \forall n, \forall m \\
& 0 \leq\left(E_{t}^{n, m, \max }-E_{t}^{n, m}\right) \perp \mu_{2, t}^{n, m} \geq 0 \quad t \in\left[t_{\text {in }}^{n, m}, t_{\text {out }}^{n, m}\right], \forall n, \forall m \\
& 0 \leq e_{t}^{n, m} \perp \mu_{3, t}^{n, m} \geq 0 \quad t \in\left[t_{\text {in }}^{n, m}, t_{\text {out }}^{n, m}\right], \forall n, \forall m \\
& 0 \leq\left(e_{t}^{n, m, \max }-e_{t}^{n, m}\right) \perp \mu_{4, t}^{n, m} \geq 0 \quad t \in\left[t_{\text {in }}^{n, m}, t_{\text {out }}^{n, m}\right], \forall n, \forall m \\
& 0 \leq\left[S_{0}^{n, m}+\sum_{t_{i n}^{n, m}}^{t}\left(E_{t}^{n, m}-e_{t}^{n, m}\right)-S^{\min }\right] \perp \mu_{5, t}^{n, m} \geq 0 \quad t \in\left[t_{\text {in }}^{n, m}, t_{\text {out }}^{n, m}\right] \\
& 0 \leq\left[S^{\max }-S_{0}^{n, m}-\sum_{t_{\text {in }}^{n, m}}^{t}\left(E_{t}^{n, m}-e_{t}^{n, m}\right)\right] \perp \mu_{6, t}^{n, m} \geq 0 \quad t \in\left[t_{\text {in }}^{n, m}, t_{\text {out }}^{n, m}\right],
\end{aligned}
$$

where $\mu_{1, t}^{n, m}$ and $\mu_{2, t}^{n, m}$ are the dual variables of constraint (15), $\mu_{3, t}^{n, m}$ and $\mu_{4, t}^{n, m}$ are the dual variables of constraint (16), $\eta_{1, t}^{n, m}$ and $\eta_{2, t}^{n, m}$ are the dual variables of constraint (17), $\zeta^{n, m}$ is the dual variable of constraint (18), $\mu_{5, t}^{n, m}$ and $\mu_{6, t}^{n, m}$ are the dual variables of constraint (19), and the symbol $\perp$ denotes that the inner product of the two vectors equals zero.

Due to the existence of non-linearities in the complementary slackness conditions of (22)-(27), they are linearized by using the Big-M method as follows:

$$
\begin{aligned}
& 0 \leq E_{t}^{n, m} \leq \mathrm{M} \times\left(1-b_{1, t}^{n, m}\right), \quad 0 \leq \mu_{1, t}^{n, m} \leq \mathrm{M} \times b_{1, t}^{n, m} \\
& 0 \leq\left(E^{n, m, \max }-E_{t}^{n, m}\right) \leq \mathrm{M} \times\left(1-b_{2, t}^{n, m}\right), 0 \leq \mu_{2, t}^{n, m} \leq \mathrm{M} \times b_{2, t}^{n, m} \\
& 0 \leq e_{t}^{n, m} \leq \mathrm{M} \times\left(1-b_{3, t}^{n, m}\right), \quad 0 \leq \mu_{3, t}^{n, m} \leq \mathrm{M} \times b_{3, t}^{n, m} \\
& 0 \leq\left(e^{n, m, \max }-e_{t}^{n, m}\right) \leq \mathrm{M} \times\left(1-b_{4, t}^{n, m}\right), \quad 0 \leq \mu_{4, t}^{n, m} \leq \mathrm{M} \times b_{4, t}^{n, m} \\
& 0 \leq\left[S_{0}^{n, m}+\sum_{t_{i n}^{n, m}}^{t}\left(E_{t}^{n, m}-e_{t}^{n, m}\right)-S^{\min }\right] \leq \mathrm{M} \times\left(1-b_{5, t}^{n, m}\right), \quad 0 \leq \mu_{5, t}^{n, m} \leq \mathrm{M} \times b_{5, t}^{n, m} \\
& 0 \leq\left[S^{\max }-S_{0}^{n, m}-\sum_{t_{i n}^{n, m}}^{t}\left(E_{t}^{n, m}-e_{t}^{n, m}\right)\right] \leq \mathrm{M} \times\left(1-b_{6, t}^{n, m}\right), \quad 0 \leq \mu_{6, t}^{n, m} \leq \mathrm{M} \times b_{6, t}^{n, m},
\end{aligned}
$$

where $b_{1, t}^{n, m} \rightarrow b_{6, t}^{n, m}$ are binary variables, and $\mathrm{M}$ is a large-enough constant. 
According to (20) and (21), one has:

$$
\begin{aligned}
& \pi_{t}^{n} E_{t}^{n, m}=\left(\mu_{1, t}^{n, m}-\mu_{2, t}^{n, m}+\eta_{1, t}^{n, m}+\zeta^{n, m}+\sum_{t}^{t_{\text {out }}^{n, m}} \mu_{5, t}^{n, m}-\sum_{t}^{t_{\text {out }}^{n, m}} \mu_{6, t}^{n, m}\right) E_{t}^{n, m} \\
& \pi_{t}^{n} e_{t}^{n, m}=\left(-\mu_{3, t}^{n, m}+\mu_{4, t}^{n, m}-\eta_{2, t}^{n, m}+\zeta^{n, m}+\sum_{t}^{t_{\text {out }}^{n, m}} \mu_{5, t}^{n, m}-\sum_{t}^{t_{\text {out }}^{n, m}} \mu_{6, t}^{n, m}\right) e_{t}^{n, m} .
\end{aligned}
$$

By subtracting (35) from (34) and using (22)-(25), one has:

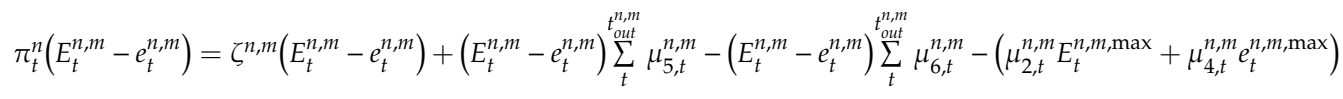

According to (26) and (27), one has:

$$
\begin{aligned}
& \sum_{t=1}^{T}\left(\left(E_{t}^{n, m}-e_{t}^{n, m}\right) \sum_{t}^{t_{\text {out }}^{n, m}} \mu_{5, t}^{n, m}\right)=\sum_{t=1}^{T}\left(\mu_{5, t}^{n, m} \sum_{t_{\text {out }}^{n, m}}^{t}\left(E_{t}^{n, m}-e_{t}^{n, m}\right)\right)=\sum_{t=1}^{T}\left(\mu_{5, t}^{n, m}\left(S^{\min }-S_{o}^{n, m}\right)\right) \\
& \sum_{t=1}^{T}\left(\left(E_{t}^{n, m}-e_{t}^{n, m}\right) \sum_{t}^{t_{\text {out }}^{n, m}} \mu_{6, t}^{n, m}\right)=\sum_{t=1}^{T}\left(\mu_{6, t}^{n, m} \sum_{t_{\text {out }}^{n, m}}^{t}\left(E_{t}^{n, m}-e_{t}^{n, m}\right)\right)=\sum_{t=1}^{T}\left(\mu_{6, t}^{n, m}\left(S^{\max }-S_{o}^{n, m}\right)\right) .
\end{aligned}
$$

Based on (37) and (38), accumulating the equation (36) over the time horizon, one can obtain:

$$
\sum_{t=1}^{T} \pi_{t}^{n}\left(E_{t}^{n, m}-e_{t}^{n, m}\right)=\sum_{t=1}^{T}\left[\zeta^{n, m}\left(E_{t}^{n, m}-e_{t}^{n, m}\right)+\mu_{5, t}^{n, m}\left(S^{\min }-S_{o}^{n, m}\right)-\mu_{6, t}^{n, m}\left(S^{\max }-S_{o}^{n, m}\right)-\left(\mu_{2, t}^{n, m} E_{t}^{n, m, m a x}+\mu_{4, t}^{n, m} e_{t}^{n, m, \max }\right)\right] .
$$

According to the strong duality theorem, the optimal solutions to the primal and the associated dual problem will yield the same objective value [30]. Therefore, substituting (18) into (39), one has:

$$
\sum_{t=1}^{T} \pi_{t}^{n}\left(E_{t}^{n, m}-e_{t}^{n, m}\right)=\xi^{n, m} S^{n, m}-\sum_{t=1}^{T}\left(\mu_{2, t}^{n, m} E_{t}^{n, m, \max }+\mu_{4, t}^{n, m} e_{t}^{n, m, \max }\right)+\sum_{t=1}^{T}\left[\mu_{5, t}^{n, m}\left(S^{\min }-S_{o}^{n, m}\right)-\mu_{6, t}^{n, m}\left(S^{\max }-S_{o}^{n, m}\right)\right]
$$

Therefore, the upper-level objective function can be reformulated as:

$$
\begin{aligned}
& {\left[a\left(\|\mathbf{R}\|_{\infty}\right)^{2}+b\|\mathbf{R}\|_{\infty}\right]-\sum_{n=1}^{N} \sum_{m=1}^{M} \sum_{t=1}^{T} \pi_{t}^{n}\left(E_{t}^{m, n}-e_{t}^{m, n}\right)=\left[a\left(\|\mathbf{R}\|_{\infty}\right)^{2}+b\|\mathbf{R}\|_{\infty}\right]+\sum_{n=1}^{N} \sum_{m=1}^{M} \xi^{n, m} S^{n, m}} \\
& -\sum_{n=1}^{N} \sum_{m=1}^{M} \sum_{t=1}^{T}\left(\mu_{2, t}^{n, m} E_{t}^{n, m, \max }+\mu_{4, t}^{n, m} e_{t}^{n, m, \max }\right)+\sum_{n=1}^{N} \sum_{m=1}^{M} \sum_{t=1}^{T}\left[\mu_{5, t}^{n, m}\left(S^{\min }-S_{o}^{n, m}\right)-\mu_{6, t}^{n, m}\left(S^{\max }-S_{o}^{n, m}\right)\right]
\end{aligned}
$$

\subsubsection{Second-Order Cone Programming}

For the non-convexity of constraints (8)-(11), we introduce the variables:

$$
\begin{array}{ll}
v_{t}^{i}=\left(V_{t}^{i}\right)^{2} & \forall t, \forall i, j \in \mathbf{S} \\
i_{t}^{i j}=\left(I_{t}^{i j}\right)^{2} & \forall t, \forall i, j \in \mathbf{S} .
\end{array}
$$

Hence, the Equations (8)-(13) in the distflow branch model can be re-written as:

$$
P_{t}^{i j}-i_{t}^{i j} R_{i j}=\sum_{k \in \omega(j)} P_{t}^{j k}+P_{t}^{j} \quad \forall t, \forall i, j \in \mathbf{S}
$$




$$
\begin{array}{cc}
Q_{t}^{i j}-i_{t}^{i j} X_{i j}=\sum_{k \in \Phi(j)} Q_{t}^{j k}+Q_{t}^{j} & \forall t, \forall i, j \in \mathbf{S} \\
v_{t}^{j}=v_{t}^{i}-2\left(R_{i j} P_{t}^{i j}+X_{i j} Q_{t}^{i j}\right)+i_{t}^{i j}\left(R_{i j}^{2}+X_{i j}^{2}\right) & \forall t, \forall i, j \in \mathbf{S} \\
i_{t}^{i j}=\frac{\left(P_{t}^{i j}\right)^{2}+\left(Q_{t}^{i j}\right)^{2}}{v_{t}^{i}} & \forall t, \forall i, j \in \mathbf{S} \\
v_{t}^{i, \min } \leq v_{t}^{i} \leq v_{t}^{i, \max } & \forall t, \forall i, j \in \mathbf{S} \\
0<i_{t}^{i j} \leq i_{t}^{i j, \max } & \forall t, \forall i, j \in \mathbf{S} .
\end{array}
$$

By applying a relaxation to (47), we obtain (50), which can be rewritten as (51):

$$
\begin{gathered}
i_{t}^{i j} \geq \frac{\left(P_{t}^{i j}\right)^{2}+\left(Q_{t}^{i j}\right)^{2}}{v_{t}^{i}} \quad \forall t, \forall i, j \in \mathbf{S} \\
\left\|\begin{array}{l}
2 P_{t}^{i j} \\
2 Q_{t}^{i j} \\
i_{t}^{i j}-v_{t}^{i}
\end{array}\right\|_{2} \leq i_{t}^{i j}+v_{t}^{i} \quad \forall t, \forall i, j \in \mathbf{S} .
\end{gathered}
$$

where $\|\cdot\|_{2}$ represents the 2-norm. The process of rewriting (8)-(13) to (44)-(46), (48), (49) and (51) is defined as an SOC relaxation [31].

\subsubsection{Single-Level MISOCP Model}

After the linearization of the bilinear terms in the objective function (7) and those in the SOCP of the distflow branch model, the original game problem is reformulated as a compact form given as follows:

$$
\text { s.t. }\left\{\begin{array}{l}
\min (41) \\
(1-6),(15-19),(20-21) \\
(28-33),(42-46),(48-49),(51)
\end{array}\right.
$$

The model in (52) is a single-level MISOCP problem, which can be efficiently solved by commercial solvers, such as CPLEX and GUROBI.

\section{Case Studies}

\subsection{Simulation Parameters}

In this section, the effectiveness and performance of the proposed game-based V2G coordination strategy is verified based on numerical simulations on a modified IEEE 33-bus distribution network with connected EV clusters, as shown in Figure 3. 


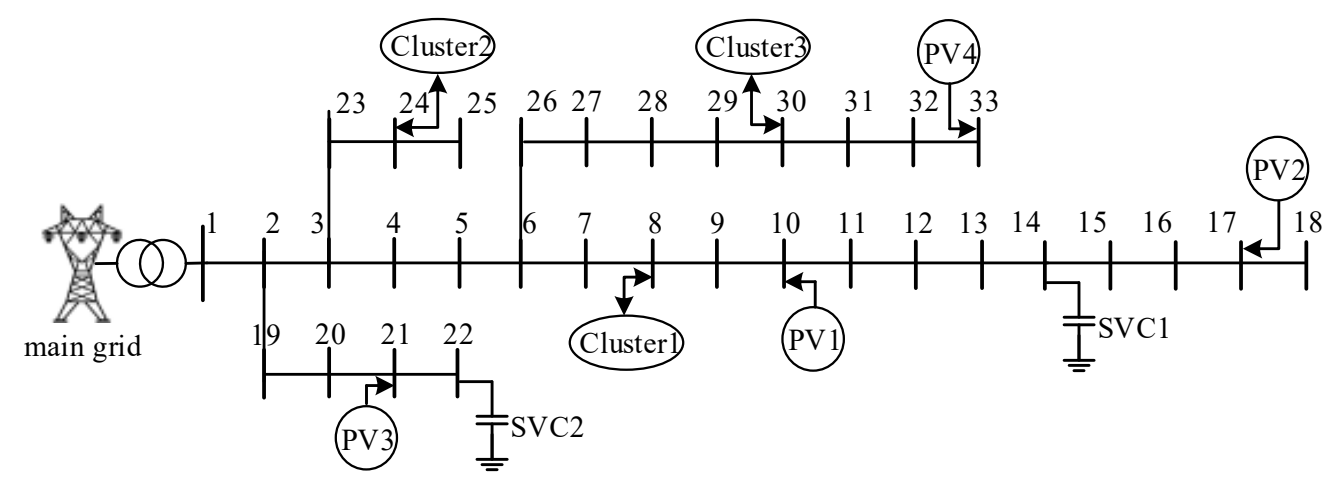

Figure 3. Modified IEEE 33-bus distribution network with EV clusters and photovoltaic (PV) generations used for simulation.

As depicted in Figure 3, buses 8, 24 and 30 individually connect a set of EV clusters; PV generation units are located at buses 10,17, 21 and 33; and SVCs are connected at buses 14 and 22. The base capacity and voltage of the distribution system are set to $10 \mathrm{MVA}$ and $12.66 \mathrm{kV}$, respectively. The normal operational range of the bus voltages is $0.95 \sim 1.05$ p.u. Each SVC has a capacity of $\pm 500 \mathrm{kVar}$, and each PV unit has a capacity of 1.1 MVA. For each bus, the load data from [29] is used as the peak load at 21:00, while the load data at other hours are obtained proportionally based on the load curve in Figure 4. The forecasted PV generation at each bus is depicted in Figure 5. The detailed data in Figures 4 and 5 are given in Appendix A.

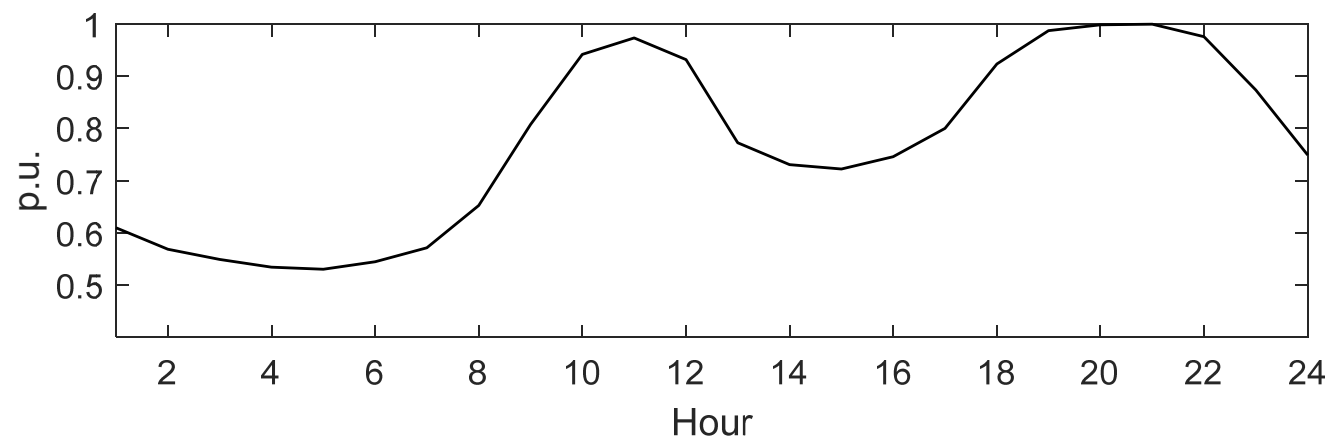

Figure 4. Normalized daily load curve.

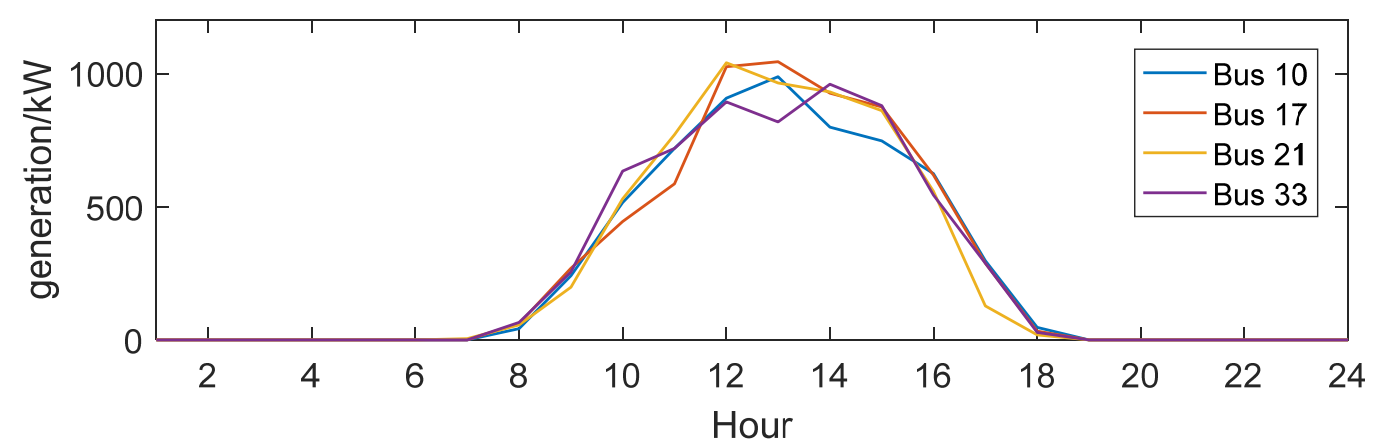

Figure 5. Hourly PV power generation.

Each EV cluster coordinates 20 EVs. Each EV has the maximum charging/discharging power of $10 \mathrm{~kW}$ and a battery capacity of $50 \mathrm{kWh}$. An EV's arrival and departure times follow a Gaussian distribution [32]. In the simulation, those two times follow $N \sim(7,0.5)$ and $N \sim(20,0.5)$ in EV Cluster 1, they follow $N \sim(6,0.5)$ and $N \sim(19,0.5)$ in EV Cluster 2 , and they follow $N \sim(8,0.5)$ and $N \sim(22,0.5)$ in EV Cluster 3, respectively. The arrival and departure times of EVs can be obtained by Monte Carlo simulation. The values of the desired energy levels $S$ of the EVs in Clusters 1,2 and 3 are randomly 
generated from the ranges $[10,20] \mathrm{kWh},[15,30] \mathrm{kWh}$ and $[20,40] \mathrm{kWh}$, respectively. It is assumed that each EV will not depart from its charging station until being fully charged. The DSO's retail price in the market is set to be 1.5 times of the wholesale price in [33], considering the DSO's construction and maintenance cost of the distribution network. The ramp cost coefficients are obtained from [28]. In this paper, all simulations are performed in MATLAB R2016A with the optimization solver of Gurobi 9.0.2.

\subsection{Simulation Results}

Figure 6 shows the transaction prices determined by the DSO in the Stackelberg game. Considering the fact that the DSO needs to offer an attractive transaction price to the EVs to encourage them to join the program, the upper and lower bounds of transaction prices are set to be 1.3 and 0.4 times of the wholesale price, respectively, which are lower than the retail electricity prices. In Figure 6, it can be seen that the transaction prices reach the upper bound before 10:00 and are between the upper and lower boundaries after 10:00. This indicates that the DSO discourages charging/discharging the EVs before 10:00 and encourages them to join the program after 10:00. From Figure 6, it is also observed that the transaction price determined by the DSO for the clusters are different after 10:00. This is due to the fact that the EV clusters, being connected at different buses in the distribution network, have different impacts on the power flows and thus will cause the DSO to generate different prices.

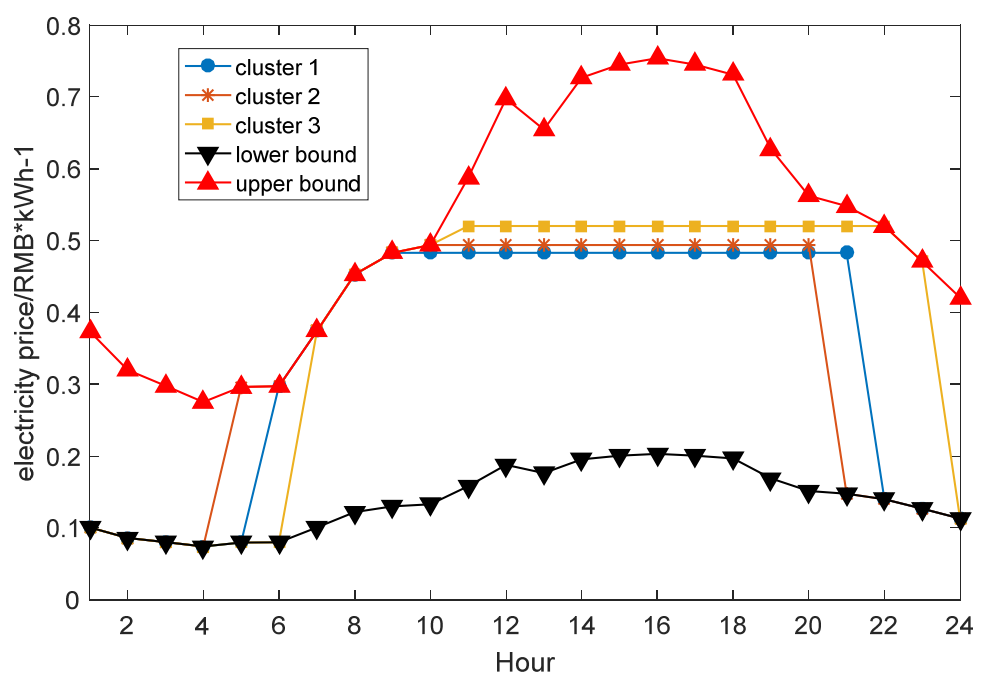

Figure 6. Hourly transaction price determined by the DSO.

Next, the impact of a cluster's coordination of the EVs' charging and discharging behaviors is analyzed. Figure 7a depicts the ramp (the change between hours) of the system net load without the EV clusters being connected, and Figure $7 \mathrm{~b}-\mathrm{d}$ depicts the charging and discharging powers of the EVs in different clusters.

In Figure 7a, the system net load has a negative ramp around 12:00 and a positive one around 17:00. The negative ramp is caused by the injection of a large amount of PV power and the reduction of load between 11:00 and 12:00 as shown in Figures 4 and 5, respectively. Similarly, the positive ramp is due to the PV power reduction and the load increase between 16:00 and 17:00. During the time periods of negative and positive ramps, the system needs to ramp down and up the generations, respectively, to maintain the power balance. The system may lack such a fast ramp or may need a high cost to ramp the generators. To address this problem, the proposed coordination strategy regulates the transaction prices (Figure 6) and uses them as incentives to encourage EV clusters' charging and discharging to contribute to the system's ramping flexibility. 


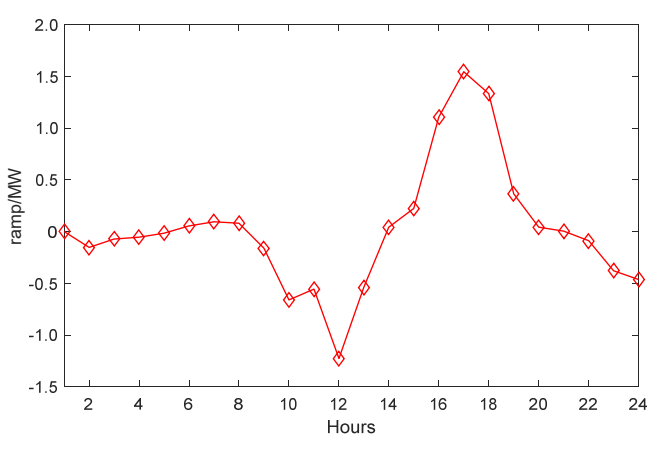

(a)

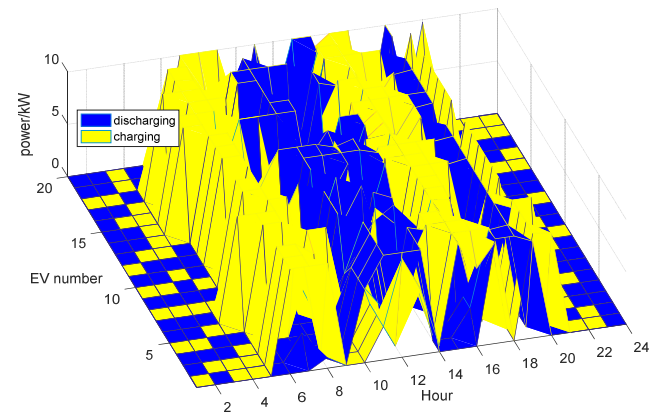

(c)

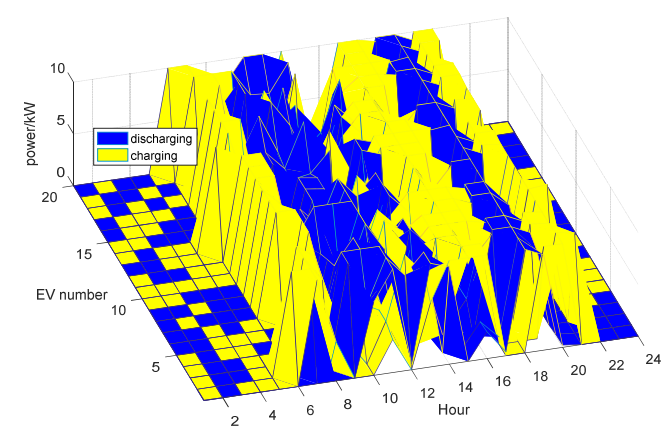

(b)

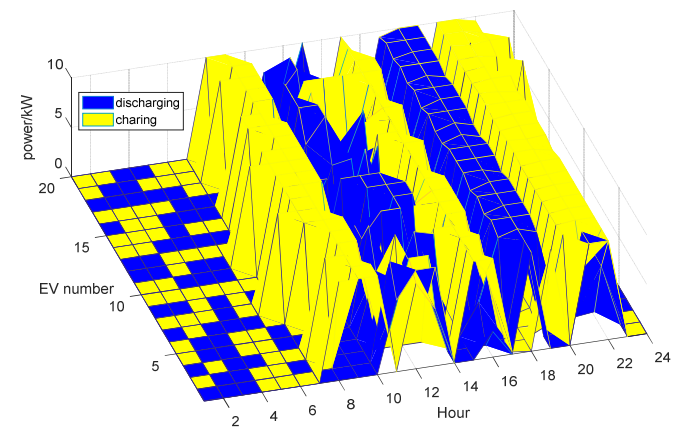

(d)

Figure 7. (a) The net-load ramp curve without EV clusters. The EVs' charging and discharging schedules in: (b) Cluster 1, (c) Cluster 2, and (d) Cluster 3.

Although the transaction prices before 10:00 hit the upper bound (see Figure 6), they are still less than the prices at other time slots. Therefore, it can be seen from Figure $7 \mathrm{~b}-\mathrm{d}$ that all the EVs choose to charge before 10:00 once connected to the grid. In addition, all the EV clusters discharge at 17:00 when the system encounters the largest positive ramp. Such discharging power can effectively alleviate the system's ramp burden caused by the impact of a PV power reduction and a load increase. In other words, the proposed coordination strategy enhances the system ramping flexibility especially at the hours with the largest net-load ramp. Similarly, Figure $7 \mathrm{~b}-\mathrm{d}$ also demonstrate that some EVs choose to charge at 12:00 when the system experiences a negative ramp.

To further study the impact of the proposed strategy on the EVs' charging costs, Table 1 compares the charging costs of the EV clusters before and after joining the program. It is obvious that the charging cost of all the EV clusters decreases when using the proposed strategy. This demonstrates that the proposed strategy is also economically sound from the perspective of EVs and therefore can attract EV owners to join the program.

Table 1. Comparison of the EV clusters' charging costs.

\begin{tabular}{cccc}
\hline Charging Costs & Cluster 1 (RMB) & Cluster 2 (RMB) & Cluster 3 (RMB) \\
\hline Under retail price & 128 & 177 & 323 \\
Proposed strategy & 118 & 171 & 290 \\
Reduction rate & $7.8 \%$ & $3.4 \%$ & $10.2 \%$ \\
\hline
\end{tabular}

Next, we studied the performance of the DSO in the coordination. Figure 8 shows the bus voltages during the operational time period. Figure 9 compares the ramp of net load at each time slot, with and without the proposed coordination. It can be seen from Figure 8 that the bus voltages are within 
the given range, which is the premise for the economic dispatch of the system. In Figure 9, when the proposed coordination is applied, the largest positive ramp decreases from 1.54 MW to 0.94 MW with a reduction rate of $39 \%$ and the largest negative ramp also decreases from $1.28 \mathrm{MW}$ to $0.90 \mathrm{MW}$, which effectively contributes ramp flexibility to the DSO. Moreover, Figure 9 shows that the net load has a relatively slower ramp before 10:00, which results from the DSO's strategy on regulating the electricity price, as analyzed in Figure 6.

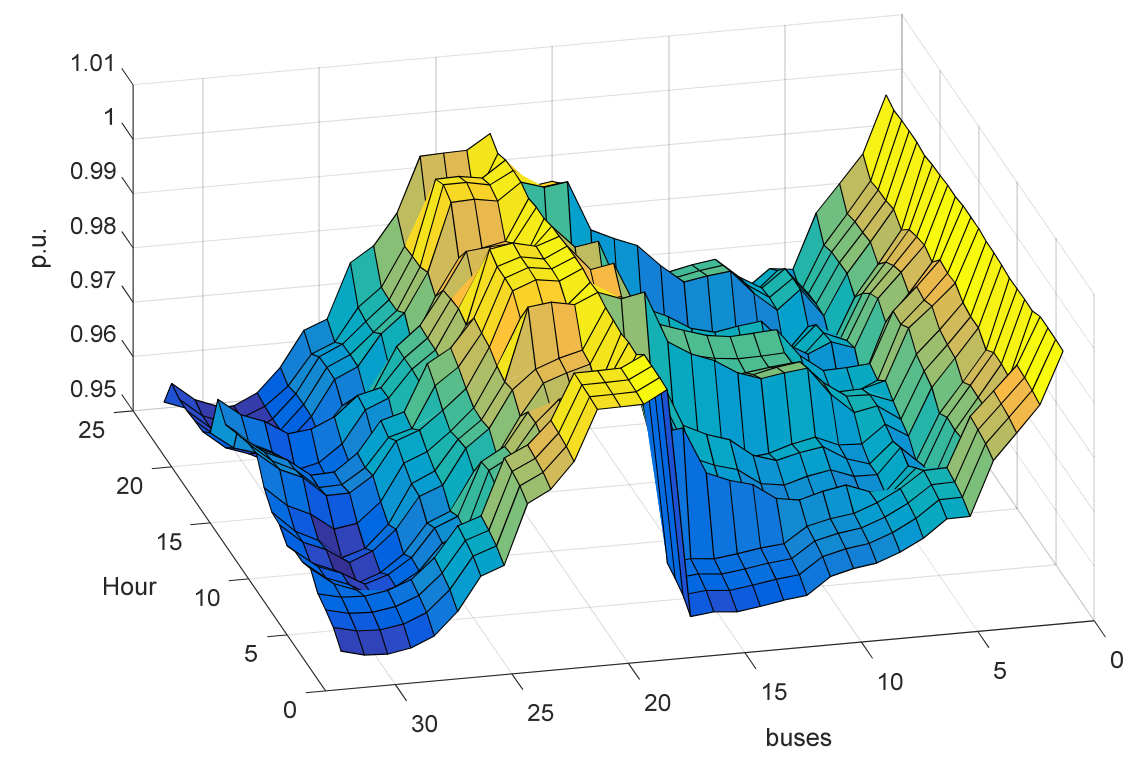

Figure 8. A 3-dimensional chart of the bus voltages in the simulated system.

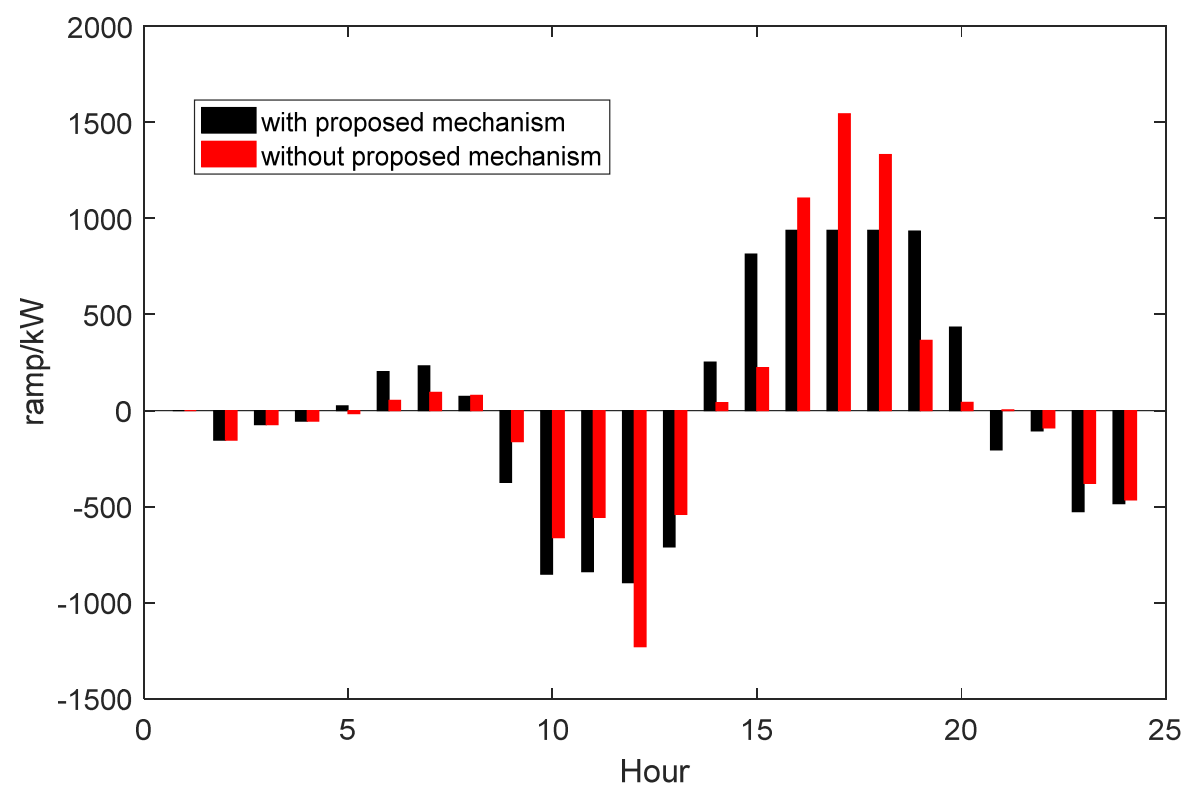

Figure 9. Comparison of the hourly ramps.

\section{Conclusions}

In this paper, a game-theory-based V2G EV cluster charging coordination strategy has been proposed to provide ramp flexibility for power systems that are integrated with large-scale variable renewable generations. The proposed strategy essentially couples EVs as flexibility providers for power systems that need ramp flexibility under high penetration levels of renewables. The strategy first models the interactions between the DSO and the EV clusters as a Stackelberg game, in which the 
DSO offers dynamic transaction prices to the EV clusters, while considering the network constraints, and the EVs reschedule their charging/discharging as a response to the dynamic prices. Next, for computational efficiency, the established bilevel model is reformulated to a single-level MISOCP problem by using KKT conditions, the strong duality theorem and SOC relaxation. Simulated results on the modified IEEE 33-bus system convincingly indicate that the proposed strategy improves the ramping flexibility of the system while reducing the charging costs of all EVs at the same time.

In this paper, the model is solved in a centralized way and the privacy issue when sharing the EVs' data is not considered. With the increasing scale of grid-connected EVs, the centralized calculation may fall into the disaster of dimensionality. Therefore, future work will be to study the privacy issue and the optimal scheduling of EVs using a distributed algorithm.

Author Contributions: Conceptualization, J.Z. and L.C.; methodology, Z.J. and L.C.; software, Z.J.; validation, L.C., L.W. and U.K.M.; formal analysis, J.Z. and L.C.; investigation, J.Z. and L.C.; resources, J.Z. and L.C.; data curation, J.Z. and L.C.; writing—original draft preparation, J.Z. and L.C.; writing-review and editing, J.Z., L.C., L.W. and U.K.M.; visualization, J.Z. and L.C.; supervision, L.C.; project administration, L.C.; funding acquisition, L.C. All authors have read and agreed to the published version of the manuscript.

Funding: This research received no external funding.

Conflicts of Interest: The authors declare no conflict of interest.

\section{Nomenclature}

A. Abbreviations

DSO

ISO

KKT

MISOCP

PV

SOC

V2G

B. Sets and Parameters

$\mathbf{N}$

M

S

T

$\mathrm{a} / \mathrm{b}$

$E^{n, m, \max } / e^{n, m, \max }$

$i_{\text {ij, } \max }$

$\stackrel{t}{S^{n, m}}$

$S_{n, m}^{\min } / S_{n, m}^{\max }$

$t_{\text {in }}^{n, m} / t_{\text {out }}^{n, m}$.

$v_{t}^{i, \min } / v_{t}^{i, \max }$

$\pi_{t}^{n, \min } / \pi_{t}^{n, \max }$

C. Variables

$E_{t}^{m, n} / e_{t}^{m, n}$

$P_{t}^{i j} / Q_{t}^{i j}$

$P_{t}^{j} / Q_{t}^{j}$

$r_{t}$

$V_{t}^{i} / I_{t}^{i j}$

$\pi_{t}^{n}$

$\mu / \eta / \zeta$

\author{
Distribution system operator \\ Independent system operator \\ Karush-Kuhn-Tucker \\ Mixed integer second-order cone programming \\ Photovoltaic \\ Second-order cone \\ Vehicle-to-grid \\ Set of EV clusters with index $n$ \\ Set of EVs with index $m$ \\ Set of distribution network buses with index $i$ and $j$ \\ Set of hours with index $t$ \\ Coefficients of peak ramp cost \\ Maximal levels of EV's charging/discharging power \\ Maximal square of the current of branch ij \\ Required net charging power \\ Lower/upper bounds of the EV battery's energy level \\ Arrival/departure times of EV $\mathrm{m}$ at cluster agent $n$ \\ Minimal/maximal squares of the voltage of bus $i$ \\ Lower/upper bounds of transaction prices between the DSO and EV cluster $n$ \\ Charging/discharging power of an EV \\ Active/reactive power flows on branch $i j$ \\ Active/reactive loads at bus $j$ \\ System ramp at time slot $t$ \\ Voltage of bus $i$ and the current of branch $i j$ \\ Transaction prices between the DSO and EV cluster $n$ \\ Dual variables in the proposed model
}




\section{Appendix A}

The hourly load and PV power data in Figures 4 and 5 are given in the following tables:

Table A1. Normalized hourly load (p.u.).

\begin{tabular}{cccccccc}
\hline Hour & Load & Hour & Load & Hour & Load & Hour & Load \\
\hline $01: 00$ & 0.6099 & $07: 00$ & 0.5715 & $13: 00$ & 0.9735 & $19: 00$ & 0.9875 \\
$02: 00$ & 0.5687 & $08: 00$ & 0.6528 & $14: 00$ & 0.9320 & $20: 00$ & 0.9989 \\
$03: 00$ & 0.5490 & $09: 00$ & 0.8078 & $15: 00$ & 0.7729 & $21: 00$ & 1.0000 \\
$04: 00$ & 0.5344 & $10: 00$ & 0.9422 & $16: 00$ & 0.7309 & $22: 00$ & 0.9760 \\
$05: 00$ & 0.5303 & $11: 00$ & 0.9735 & $17: 00$ & 0.7223 & $23: 00$ & 0.8740 \\
$06: 00$ & 0.5447 & $12: 00$ & 0.9321 & $18: 00$ & 0.7461 & $24: 00$ & 0.7491 \\
\hline
\end{tabular}

Table A2. Hourly PV power $(\mathrm{kW})$.

\begin{tabular}{cccccccccc}
\hline Hour & PV1 & PV2 & PV3 & PV4 & Hour & PV1 & PV2 & PV3 & PV4 \\
\hline $01: 00$ & 0 & 0 & 0 & 0 & $13: 00$ & 988.50 & 1045.20 & 964.80 & 818.40 \\
$02: 00$ & 0 & 0 & 0 & 0 & $14: 00$ & 799.50 & 927 & 931.80 & 960.30 \\
$03: 00$ & 0 & 0 & 0 & 0 & $15: 00$ & 747.30 & 875.10 & 861 & 879.90 \\
$04: 00$ & 0 & 0 & 0 & 0 & $16: 00$ & 624.30 & 619.50 & 558 & 543.90 \\
$05: 00$ & 0 & 0 & 0 & 0 & $17: 00$ & 297.90 & 288.60 & 127.80 & 288.60 \\
$06: 00$ & 0 & 0 & 0 & 0 & $18: 00$ & 47.40 & 33 & 18.90 & 28.50 \\
$07: 00$ & 0 & 0 & 4.80 & 0 & $19: 00$ & 0 & 0 & 0 & 0 \\
$08: 00$ & 42.60 & 61.50 & 56.70 & 66.30 & $20: 00$ & 0 & 0 & 0 & 0 \\
$09: 00$ & 241.20 & 269.70 & 198.60 & 255.30 & $21: 00$ & 0 & 0 & 0 & 0 \\
$10: 00$ & 515.70 & 444.60 & 529.80 & 633.90 & $22: 00$ & 0 & 0 & 0 & 0 \\
$11: 00$ & 719.10 & 586.50 & 771.00 & 719.10 & $23: 00$ & 0 & 0 & 0 & 0 \\
$12: 00$ & 908.10 & 1026.30 & 1040.70 & 894.00 & $24: 00$ & 0 & 0 & 0 & 0 \\
\hline
\end{tabular}

\section{References}

1. Administration, U.E.I. Annual Energy Outlook 2019: With Projections to 2050. 2020. Available online: https://www.eia.gov/outlooks/aeo/pdf/aeo2019.pdf (accessed on 1 August 2020).

2. Kwon, H.; Park, J.-K.; Kim, D.; Yi, J.; Park, H. A Flexible Ramping Capacity Model for Generation Scheduling with High Levels of Wind Energy Penetration. Energies 2016, 9, 1040. [CrossRef]

3. Che, L.; Liu, X.; Li, Z. An Intra-interval Security Risk Regarding Regulation Burden Due to Wind Variation in High-Wind-Penetrated Power Systems. IEEE Trans. Power Syst. 2018, 33, 3213-3216. [CrossRef]

4. Badal, F.R.; Das, P.; Sarker, S.K.; Das, S.K. A Survey on Control Issues in Renewable Energy Integration and Microgrid. Prot. Control Mod. Power Syst. 2019, 4, 8. [CrossRef]

5. CAISO. What the Duck Curve Tells Us about Managing a Green Grid. 2020. Available online: https: //www.caiso.com/Documents/FlexibleResourcesHelpRenewables_FastFacts.pdf (accessed on 9 August 2020).

6. Chalise, S.; Atia, H.R.; Poudel, B.; Tonkoski, R. Impact of Active Power Curtailment of Wind Turbines Connected to Residential Feeders for Overvoltage Prevention. IEEE Trans. Sustain. Energy 2016, 7, 471-479. [CrossRef]

7. Kalantari, A.; Galiana, F.D. The impact of wind power variability and curtailment on ramping requirements. In Proceedings of the 2010 IEEE/PES Transmission and Distribution Conference and Exposition: Latin America (T\&D-LA), Sao Paulo, Brazil, 8-10 November 2010; pp. 133-138.

8. Bae, H.; Tsuji, T.; Oyama., T.; Uchida, K. Frequency regulation method with congestion management using renewable energy curtailment. In Proceedings of the 2016 IEEE Power and Energy Society General Meeting (PESGM), Boston, MA, USA, 17-21 July 2016; pp. 1-5.

9. Wang, Y.; Lou, S.; Wu, Y.; Wang, S. Flexible Operation of Retrofitted Coal-Fired Power Plants to Reduce Wind Curtailment Considering Thermal Energy Storage. IEEE Trans. Power Syst. 2020, 35, 1178-1187. [CrossRef] 
10. Zhang, X.; Che, L.; Shahidehpour, M.; Alabdulwahab, A.; Abusorrah, A. Electricity-Natural Gas Operation Planning With Hourly Demand Response for Deployment of Flexible Ramp. IEEE Trans. Sustain. Energy 2016, 7, 996-1004. [CrossRef]

11. Chen, X.; Kang, C.; O’Malley, M.; Xia, Q.; Bai, J.; Liu, C.; Sun, R.; Wang, W.; Li, H. Increasing the Flexibility of Combined Heat and Power for Wind Power Integration in China: Modeling and Implications. IEEE Trans. Power Syst. 2015, 30, 1848-1857. [CrossRef]

12. Richter, M.; Möllenbruck, F.; Obermüller, F.; Knaut, A.; Weiser, F.; Lens, H.; Lehmann, D. Flexibilization of steam power plants as partners for renewable energy systems. In Proceedings of the 2016 Power Systems Computation Conference (PSCC), Genoa, Italy, 20-24 June 2016; pp. 1-8.

13. Hu, J.; Wen, F.; Wang, K.; Huang, Y.; Salam, M. Simultaneous Provision of Flexible ramping product and demand relief by interruptible loads considering economic incentives. Energies 2018, 11, 46. [CrossRef]

14. Sheha, M.; Mohammadi, K.; Powell, K. Solving the duck curve in a smart grid environment using a non-cooperative game theory and dynamic pricing profiles. Energy Convers. Manag. 2020, 11, 3102. [CrossRef]

15. Nguyen, H.K.; Khodaei, A.; Han, Z. Distributed algorithms for peak ramp minimization problem in smart grid. In Proceedings of the 2016 IEEE International Conference on Smart Grid Communications (SmartGridComm), Sydney, NSW, Australia, 6-9 November 2016; pp. 174-179.

16. Abrishambaf, O.; Faria, P.; Vale, Z. Ramping of Demand Response Event with Deploying Distinct Programs by an Aggregator. Energies 2020, 13, 1389. [CrossRef]

17. Mediwaththe, C.P.; Smith, D.B. Game-Theoretic Electric Vehicle Charging Management Resilient to Non-Ideal User Behavior. IEEE Trans. Intell. Transp. Syst. 2018, 19, 3486-3495. [CrossRef]

18. Vagropoulos, S.I.; Bakirtzis, A.G. Optimal Bidding Strategy for Electric Vehicle Aggregators in Electricity Markets. IEEE Trans. Power Syst. 2013, 28, 4031-4041. [CrossRef]

19. Annamraju, A.; Nandiraju, S. Coordinated control of conventional power sources and PHEVs using jaya algorithm optimized pid controller for frequency control of a renewable penetrated power system. Prot. Control Mod. Power Syst. 2019, 4, 28. [CrossRef]

20. Ko, K.S.; Han, S.; Sung, D.K. A New Mileage Payment for EV Aggregators with Varying Delays in Frequency Regulation Service. IEEE Trans. Smart Grid 2018, 9, 2616-2624. [CrossRef]

21. Haghi, H.V.; Qu, Z. A Kernel-Based Predictive Model of EV Capacity for Distributed Voltage Control and Demand Response. IEEE Trans. Smart Grid 2018, 9, 3180-3190. [CrossRef]

22. Cheng, L.; Chang, Y.; Huang, R. Mitigating Voltage Problem in Distribution System with Distributed Solar Generation Using Electric Vehicles. IEEE Trans. Sustain. Energy 2015, 6, 1475-1484. [CrossRef]

23. Luo, X.; Xia, S.; Chan, K.W.; Lu, X. A Hierarchical Scheme for Utilizing Plug-in Electric Vehicle Power to Hedge Against Wind-Induced Unit Ramp Cycling Operations. IEEE Trans. Power Syst. 2018, 33, 55-69. [CrossRef]

24. Zhang, B.; Kezunovic, M. Impact on Power System Flexibility by Electric Vehicle Participation in Ramp Market. IEEE Trans. Smart Grid 2016, 7, 1285-1294. [CrossRef]

25. Zhang, X.; Hu, J.; Wang, H.; Wang, G.; Chan, K.W.; Qiu, J. Electric Vehicle Participated Electricity Market Model Considering Flexible Ramping Product Provisions. IEEE Trans. Ind. Appl. 2020. [CrossRef]

26. Massier, T.; Melo, D.F.R. Integration of renewables in Singapore: Ramp rate support using electric vehicles. In Proceedings of the 2017 IEEE Innovative Smart Grid Technologies-Asia (ISGT-Asia), Auckland, New Zealand, 4-7 December 2017; pp. 1-6.

27. Ko, K.S.; Sung, D.K. The Effect of EV Aggregators with Time-Varying Delays on the Stability of a Load Frequency Control System. IEEE Trans. Power Syst. 2018, 33, 669-680. [CrossRef]

28. Nguyen, H.K.; Khodaei, A.; Han, Z. Incentive Mechanism Design for Integrated Microgrids in Peak Ramp Minimization Problem. IEEE Trans. Smart Grid 2018, 9, 5774-5785. [CrossRef]

29. Baran, M.E.; Wu, F.F. Network reconfiguration in distribution systems for loss reduction and load balancing. IEEE Trans. Power Del. 1989, 4, 1401-1407. [CrossRef]

30. Bruninx, K.; Pandžić, H.; Cadre, H.L.; Delarue, E. On the Interaction between Aggregators, Electricity Markets and Residential Demand Response Providers. IEEE Trans. Power Syst. 2020, 35, 840-853. [CrossRef]

31. Huang, S.; Wu, Q.; Wang, J.; Zhao, H. A Sufficient Condition on Convex Relaxation of AC Optimal Power Flow in Distribution Networks. IEEE Trans. Power Syst. 2017, 32, 1359-1368. [CrossRef] 
32. Chen, X.; Leung, K. Non-Cooperative and Cooperative Optimization of Scheduling with Vehicle-to-Grid Regulation Services. IEEE Trans. Veh. Technol. 2020, 69, 114-130. [CrossRef]

33. Wang, H.; Huang, J. Incentivizing Energy Trading for Interconnected Microgrids. IEEE Trans. Smart Grid 2018, 9, 2647-2657. [CrossRef]

(C) 2020 by the authors. Licensee MDPI, Basel, Switzerland. This article is an open access article distributed under the terms and conditions of the Creative Commons Attribution (CC BY) license (http://creativecommons.org/licenses/by/4.0/). 